Redina Ljumanovic Johannes A. Langendijk

Otto S. Hoekstra

Dirk L. Knol

C. René Leemans

Jonas A. Castelijns

\section{Pre- and post-radiotherapy MRI results as a predictive model for response in laryngeal carcinoma}

Received: 8 November 2007

Revised: 16 February 2008

Accepted: 22 March 2008

Published online: 20 May 2008

(C) The Author(s) 2008

R. Ljumanovic $(\bowtie) \cdot J$. A. Castelijns

Department of Radiology, VU

University Medical Center,

De Boelelaan 1117, $1081 \mathrm{HV}$

Amsterdam,

P.O. Box 7057, 1007 MB

Amsterdam, The Netherlands

e-mail: Redina.Ljumanovic@vumc.nl

Tel.: +31-20-4440363

Fax: +31-20-4442831

\section{J. A. Langendijk}

Department of Radiation Oncology, University Medical Center Groningen, Groningen, The Netherlands

\section{O. S. Hoekstra}

Department of Nuclear Medicine and PET research, VU University Medical Center,

Amsterdam, The Netherlands

\section{L. Knol}

Department of Clinical Epidemiology

and Biostatistics, VU University

Medical Center,

Amsterdam, The Netherlands

\author{
C. R. Leemans \\ Department of Otolaryngology Head \\ and Neck Surgery, VU University \\ Medical Center, \\ Amsterdam, The Netherlands
}

\begin{abstract}
The purpose was to determine if pre-radiotherapy (RT) and/or post-radiotherapy magnetic resonance (MR) imaging can predict response in patients with laryngeal carcinoma treated with RT. Pre- and post-RT MR examinations of 80 patients were retrospectively reviewed and associated with regard to local control. Pre-RT MR imaging parameters such as tumor involvement of specific laryngeal anatomic subsites including laryngeal cartilages and post-RT changes, i.e., complete resolution of the tumor or focal mass/asymmetric obliteration of laryngeal tissue and signal pattern on T2-weighted images, were evaluated. Local control was defined as absence of a recurrence at the primary site for 2 years. Local control rates based on pretreatment MR findings were $73 \%$ for low preRT risk-profile and $29 \%$ for high pre-
\end{abstract}

RT risk-profile patients ( $p=0.0001)$. Based on posttreatment MR findings, local control rates were $100 \%$ score 1 , $64 \%$ score 2 , and $4 \%$ score 3 ( $\mathrm{p}<$ 0.0001). Using post-RT T2-weighted images, significant association was found between differences in signal pattern and local control: 77\% hypointense, $54 \%$ isointense and $15 \%$ hyperintense lesions $(p<0.001)$. Differences between means of delay of post-MRI examination were significantly associated with regard to local control $(\mathrm{p}=0.003)$; recurrent tumors followed 5 months after RT were more easily detectable on MRI than recurrent tumors within 4 months after RT. Sensitivity, specificity, accuracy, negative and positive predictive values of post-RT score 3 were $96 \%, 76 \%, 83 \%$, $98 \%$ and $66 \%$. Pre- and post-RT MRI evaluation of the larynx can identify patients at high risk for developing local failure.

Keywords MR imaging · Laryngeal neoplasm - Radiation oncology · Response to treatment

\section{Introduction}

Imaging modalities like computed tomography $(\mathrm{CT})$ and magnetic resonance (MR) imaging are increasingly used in follow-up of head and neck tumors. Promising results have also been achieved for differentiation between residual or recurrent head and neck tumors and postoperative or postradiation changes with diffusion-weighted echo-planar MR imaging [1]. However, the difference in pathology and type of treatment and postradiation changes would definitely affect the ADC value. The ability to provide an accurate pretreatment prediction of response may offer a possible opportunity to modify the initial treatment regimen to improve clinical outcome. 
The preliminary studies suggest that with $\mathrm{CT}$ and MR imaging it is possible to identify patients treated with primary radiotherapy (RT) for squamous cell head and neck cancer that are at increased risk for local recurrence [2-11].

Residual or recurrent tumor after treatment can be difficult to detect by physical examination due to the radiation effects producing varying degrees of persistent mucositis, edema and fibrosis and cartilage necrosis. Approximately $50 \%$ of patients with severe edema or necrosis following radiotherapy will have a recurrence [12]. The need for biopsy itself can present a dilemma as this may exacerbate postradiotherapy changes. Familiarization with the expected imaging changes following radiotherapy allows accurate evaluation of imaging studies and may prevent misinterpretation of posttreatment changes as recurrent disease. Baseline imaging studies done specifically for follow-up of the primary site are usually obtained 3 to 4 months after completion of radiotherapy $[13,14]$. Until now, most studies focused on posttreatment $\mathrm{CT}$ appearances of the irradiated larynx and indicated that post-RT CT evaluations can identify patients at increased risk for developing local recurrence and proved to be a better prognosticator than the pretreatment $\mathrm{CT}$ risk profiles [15-17]. The expected appearance of the irradiated larynx at MR imaging has received limited attention in the radiological literature [18]. Advantages of MR imaging include superior soft tissue contrast and exact delineation of tumor margins in arbitrary planes. Moreover, MR imaging may identify fibrosis showing low signal intensity (SI) in T1- and T2-weighted MR images, and consequently may allow differentiation between tumor and fibrosis $[19,20]$. On T2-weighted MR images signal intensity of tumors is generally higher than signal intensity of fibrosis $[19,21,22]$.

The purpose of this retrospective study is to determine if pre-RT and/or post-RT MR imaging can predict the risk for local failure in patients with laryngeal carcinoma treated with irradiation alone or combined with chemotherapy.

\section{Materials and methods}

Patient selection

The hospital charts of 160 patients who underwent radiation therapy with curative intent for squamous cell carcinoma of the larynx between June 1995 and May 2003 were retrospectively reviewed. At least 24 months of follow-up after completion of RT was required. Out of these 160 patients, $80(50 \%)$ were eligible for this study, including 63 men (79\%; mean age, 63 years; range, 3982 years) and 17 women ( $21 \%$; mean age, 60 years; range, 45-71 years). The major reasons for exclusion were less than 24 months' follow-up after RT, patients with a previous history of laryngeal cancer or other malignant diseases in the head and neck region, and lack of suitable
MR imaging studies. Stage of disease in all patients was appraised clinically according to the UICC recommendations [23]. The institutional review board waived patient informed consent since all images were acquired for clinical purposes and were considered as existing data documents. The pretreatment tumor characteristics are listed in Table 1.

All patients were treated with primary radiation therapy with a mean total dose of $67 \mathrm{~Gy}$ (median, $70 \mathrm{~Gy}$; range, 60$70 \mathrm{~Gy}$ ) using 2.0 to $2.5 \mathrm{~Gy}$ per fraction. Twelve patients $(15 \%)$ received neoadjuvant chemotherapy prior to radiation therapy. Follow-up included indirect laryngoscopy at 2-month intervals in the first 2 years after finishing radiation therapy and every 4-6 months thereafter, supplemented in case of suspicion by direct laryngoscopy under anesthesia with biopsies if indicated. No patient was lost to follow-up. The follow-up period was designated as the total time of follow-up ending either at local recurrence or at last patient contact without local recurrence with a minimum of 2 years (mean follow-up time, 23.7 months; range, 4.5-107.7 months).

\section{MR imaging}

MR imaging was performed with a $1.0-\mathrm{T}$ or $1.5-\mathrm{T}$ MR machine (Siemens-Impact or Vision, Erlangen, Germany). A neck surface coil (Medical Advances, Milwaukee, WI) was

Table 1 Pretreatment tumor characteristics $(n=80)$

\begin{tabular}{|c|c|c|c|}
\hline \multirow[t]{2}{*}{ Variable } & \multicolumn{3}{|c|}{ Number of patients } \\
\hline & Supraglottic site & Glottic site & $\%$ of total \\
\hline \multicolumn{4}{|l|}{$\mathrm{T}$ classification } \\
\hline $\mathrm{T} 1$ & & 6 & 7 \\
\hline $\mathrm{T} 2$ & 12 & 32 & 55 \\
\hline T3 & 12 & 3 & 19 \\
\hline $\mathrm{T} 4$ & 8 & 7 & 19 \\
\hline \multicolumn{4}{|l|}{$\mathrm{N}$ classification } \\
\hline No & 23 & 47 & 88 \\
\hline N1 & 8 & 1 & 11 \\
\hline $\mathrm{N} 2$ & 1 & & 1 \\
\hline \multicolumn{4}{|c|}{ Vocal cord mobility (both sides) } \\
\hline Normal & 23 & 19 & 53 \\
\hline Impaired & 6 & 23 & 36 \\
\hline Fixed & 3 & 6 & 11 \\
\hline \multicolumn{4}{|l|}{ Histopathology } \\
\hline Well differentiated & 3 & 15 & 23 \\
\hline Moderately differentiated & 22 & 26 & 60 \\
\hline Poorly differentiated & 7 & 6 & 16 \\
\hline Undifferentiated & & 1 & 1 \\
\hline \multicolumn{4}{|l|}{ Primary treatment } \\
\hline Radiotherapy & 22 & 46 & 85 \\
\hline Radiochemotherapy & 10 & 2 & 15 \\
\hline
\end{tabular}


used for higher spatial resolution. Pre-RT MR images with standard spin-echo T1-weighted (repetition time ms/echo time ms, 310-800/15), fast T2-weighted (2,200-4,550/90-98), and contrast-enhanced T1-weighted images (310-800/15) were available. Post-RT MR images were obtained in a similar plane to that of the pre-RT non-contrast and contrast-enhanced images. Other technical factors included a slice thickness of 3 $7 \mathrm{~mm}$ with a 1-mm intersection gap, and each acquisition lasted approximately 2-5 min.

Two authors (JAC, RLJ) working in consensus, with respectively 13 and 4 years of experience of head and neck MR imaging, who were unaware of the patients' clinical findings retrospectively performed the imaging assessment of all MRI studies. On pre-RT MRI examinations, each tumor was assigned a low- or high-risk profile for local failure after RT, based on previously determined specific $\mathrm{CT}$ and MR imaging parameters [2, 11]. Patients with supraglottic carcinoma were considered to be at high risk if tumor invasion in the pre-epiglottic space in combination with abnormal signal intensity of thyroid adjacent to the anterior commissure and/or cricoid cartilage was present [2]. A high-risk profile was attributed to a glottic carcinoma if the tumor extended to the hypopharynx or if a tumor mass demonstrated an intermediate T2 signal in cartilage similar to tumor intensity [11]. In 14 patients the pretreatment MRI study was not available. Determination of the pretreatment risk profile in the patients who received RT

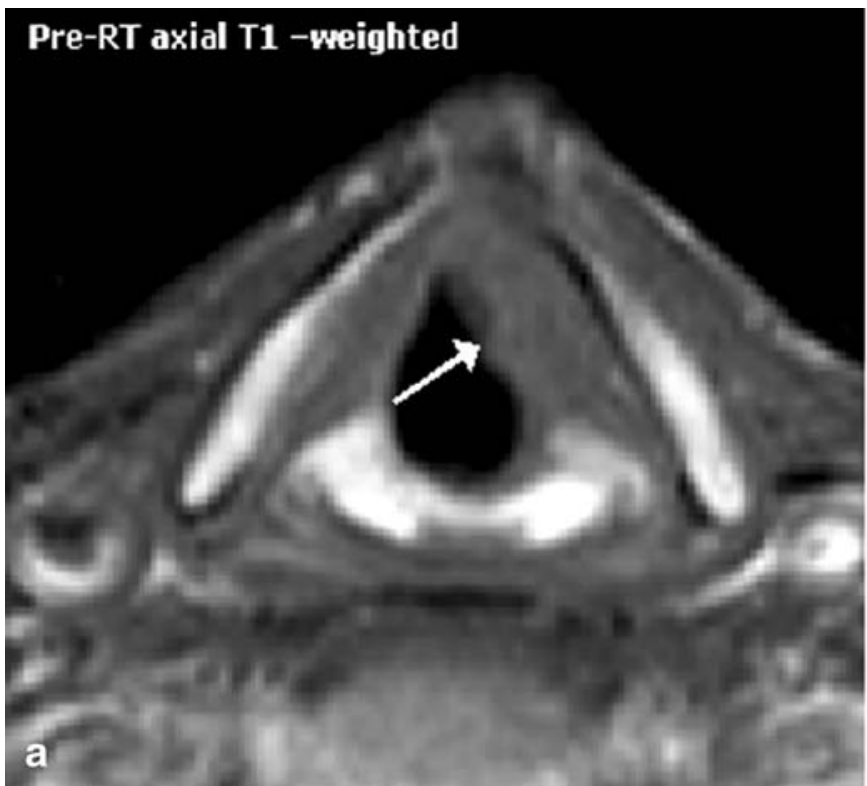

Fig. 1 Clinical stage T2 glottic carcinoma in a 63-year-old patient with low pre-treatment MR risk profile. (A) Pre-treatment axial T1weighted MR image at the level of the true vocal cord shows a mass (arrow) with intermediate signal intensity in the left cord. (B) Image combined with neoadjuvant chemotherapy was based on a MR imaging before initiation of chemotherapy.

The post-RT MRI examinations were evaluated for changes using a three-point score $[15,16]$ : $1=$ expected post-RT changes, i.e., complete resolution of the tumor at the primary site and symmetrically appearing laryngeal and hypopharyngeal tissues (Fig. 1);2 = focal mass with a maximal diameter of less than $1 \mathrm{~cm}$ and/or asymmetric obliteration of laryngeal tissue planes (Fig. 2); $3=$ focal mass with a maximal diameter of more than $1 \mathrm{~cm}$, or less than 50\% estimated tumor volume reduction (Fig. 3). According to preliminary experience with CT as imaging method [14-16], this post-RT MRI scoring system can be similarly interpreted as: 1 = likely to achieve permanent local control, no evidence of disease; 2 = indeterminate, suspicious for local failure or treatment complication; $3=$ indicated high likelihood of local failure. The MRI examinations used for analysis were performed as part of routine follow-up $(n=51)$ or because of certain symptomatology, potentially indicating local recurrence $(n=29)$.

The signal patterns of posttreatment changes were observed on the T2-weighted images of post-RT MRI examinations, but enhancement on post-contrast T1weighted images was evaluated if interpretation of T2 images was not optimal (Figs. 4, 5 and 6). The MR imaging criteria for recurrent or residual tumor are an enlarging enhancing infiltrating mass that is of intermediate to high signal intensity on T2-weighted MR images [18, 24]. Areas

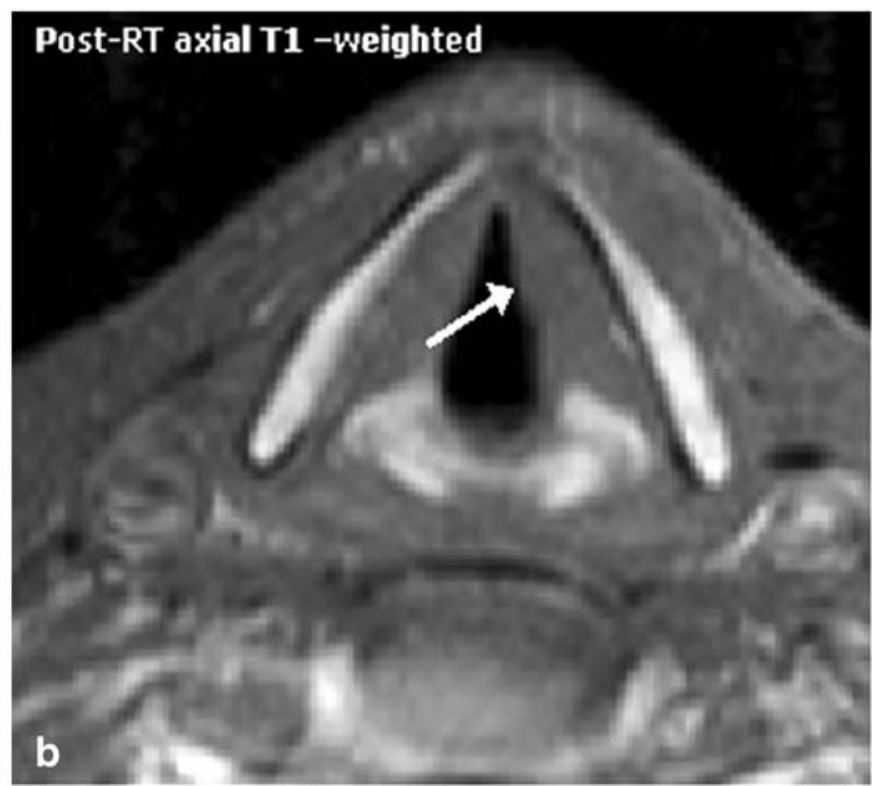

obtained 4 months post-RT (after radiation therapy) at the same level. There is complete resolution of the tumor (arrowheads). The laryngeal tissue appears symmetric and post-RT MR score is 1 . Local recurrence was not documented within 24 months after RT 
with very high signal intensity (equivalent to water) in T2weighted images were regarded as necrosis and not included in this study. The hypointense areas were considered as fibrotic changes [18].

All 80 patients in this study had undergone at least one follow-up examination after radiation therapy. The 80 follow-up MR scans were obtained between 1 and 16 months after completion of radiotherapy (in 7 patients more than 9 months). The mean time between completion of radiotherapy and MRI was 5 months (median, 4 months).

\section{Statistical analyses}

The endpoint used in the statistical analyses was local control at 2 years. Intervals were measured from the first day of radiation therapy to the date of failure or the date of last follow-up. The Kaplan-Meier method was used to estimate curves and differences between curves we tested by means of the log-rank test. Calculation of risk ratios and simultaneous testing of pre-RT and post-RT MRI scores were performed by Cox proportional hazards analysis. In the analysis, post-RT MRI score was considered linearly related with the log (hazard).

Tumor lesions on T2-weighted MR images after primary treatment were classified into three groups, hypointense, isointense/mixed and hyperintense, and were associated using Cox regression model with regard to local control. Differences in the means of delay of post-RT MRI examination were tested with a one-way analysis of variance.

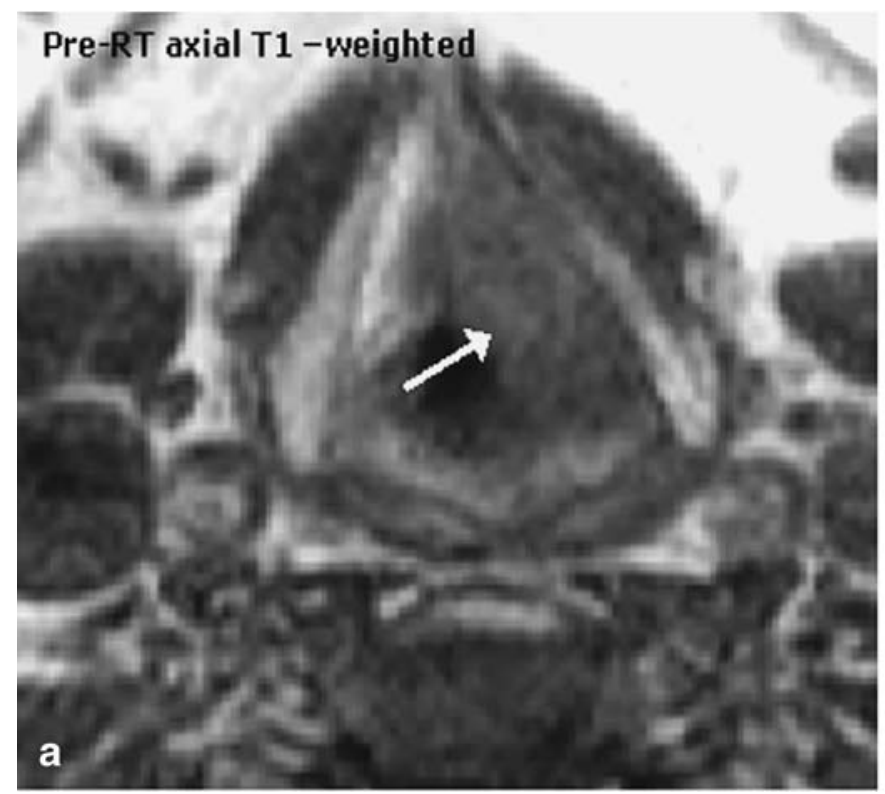

Fig. 2 Clinical stage T1b glottic carcinoma in a 62-year-old patient with high pre-treatment MR risk profile. (A) Pre-treatment axial T1weighted MR image at the level of the true vocal cord shows a mass (arrow) with intermediate signal intensity in the left cord. (B) MR
The sensitivity, specificity, accuracy, and negative and positive predictive values of follow-up MR imaging for detection of local failure (score of 3 ) were calculated.

A p-value $<0.05$ was designated for determining significant differences. The SPSS version 11.0 (SPSS, Chicago, IL) software package was used for analysis.

\section{Results}

The mean age at the time of radiotherapy was 63 years (range, 39-82 years). There were no statistically significant age-related differences between the male and female patients (Mann-Whitney test, $\mathrm{p}=0.3$ ), which matched the sex ratio for laryngeal carcinoma.

A total number of 38 out 80 patients (48\%) developed local recurrence, which was confirmed by histopathological examination. Six patients $(6 / 12 ; 50 \%)$ were treated with chemoradiation and 32 patients $(32 / 68 ; 47 \%)$ with irradiation alone. No significant difference was found between these two groups. Thirty-four out of these 38 patients (89\%) underwent salvage (partial) laryngectomy, 3 patients refused salvage treatment, and 1 patient died suddenly after recurrent tumor was diagnosed.

\section{Pre-RT MRI risk profile}

In 66 out of 80 patients ( $83 \%)$ the pretreatment MR scans were available for review. The pretreatment MR imaging

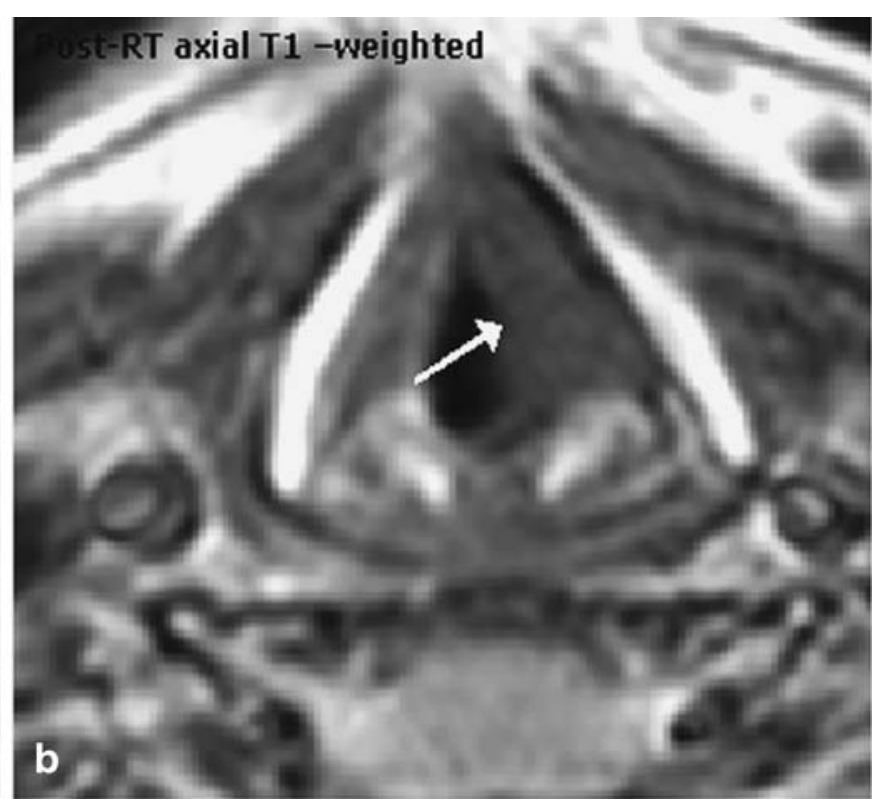

image at the same level, obtained at 7 months post-RT, shows that the tumor has regressed, but there is asymmetric obliteration of the fatty tissue at the left side without obvious focal mass. Post-RT MR score is 2 . There was no clinical evidence of disease 


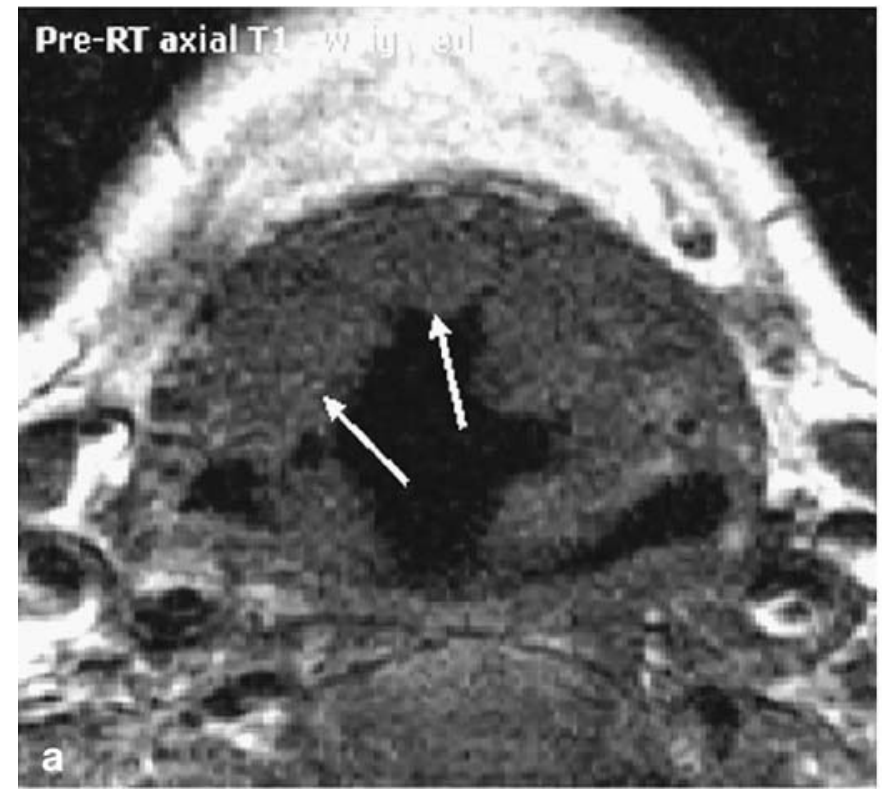

Fig. 3 Clinical stage T3 supraglottic carcinoma in a 64-year-old patient with high pre-treatment MR risk profile. (A) Pre-treatment axial T1-weighted MR image demonstrates a tumor mass (arrows) with intermediate signal intensity on the left and right sides. (B) MR

high-risk parameters, as defined in the previous section, are illustrated in Table 2. There were 26 patients with supraglottic carcinoma (low-risk group, $\mathrm{n}=14$; high-risk group, $\mathrm{n}=12$ ) and 40 patients with glottic carcinoma (lowrisk group, $\mathrm{n}=24$; high-risk group, $\mathrm{n}=16$ ). Figure 7

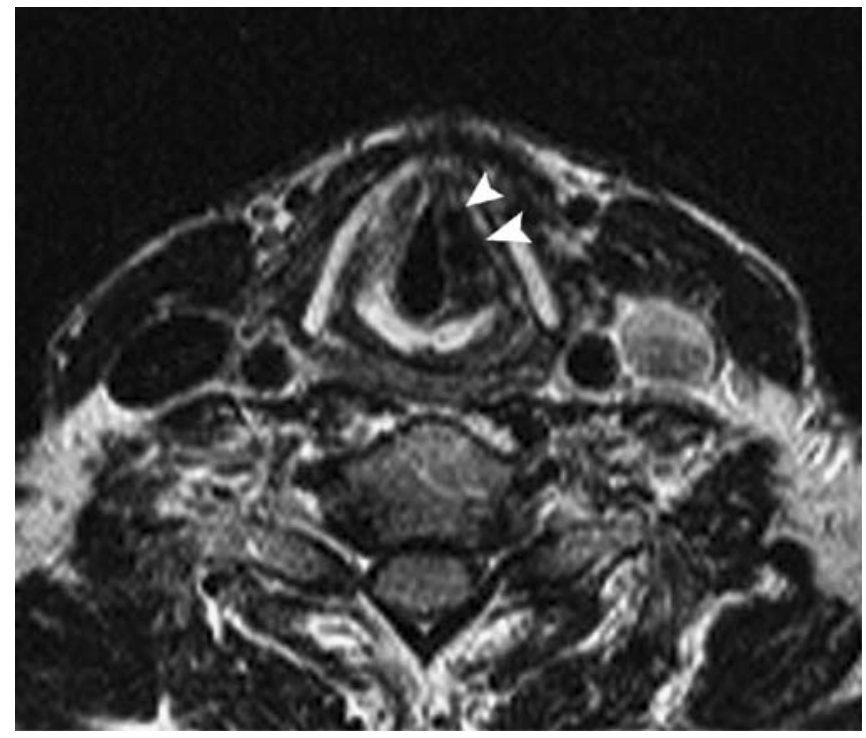

Fig. 4 Clinical stage T2 glottic carcinoma in an 80-year-old patient. Posttreatment axial T2-weighted MR image, obtained 11 months after RT, at the level of the true vocal cord demonstrates a tumor mass (arrowheads) with low signal intensity. The tumor was considered as a hypointense tumor, which did not locally recur within 24 months after RT

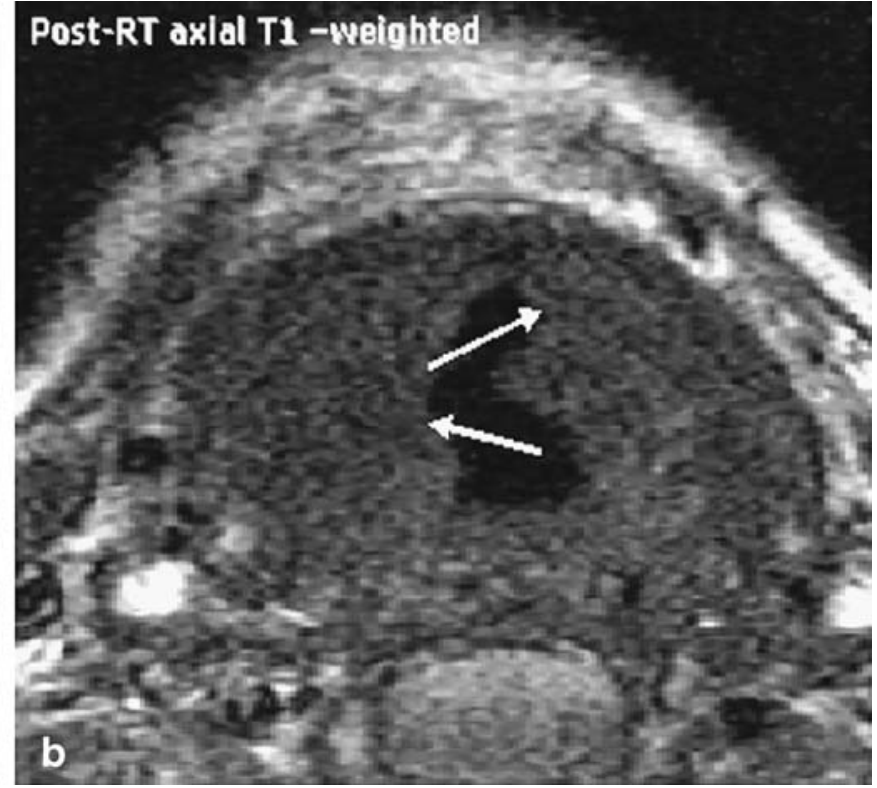

image obtained 6 months after RT shows a focal mass (arrows) a maximal diameter greater than $1 \mathrm{~cm}$ in the original tumor bed. This image had MR score of 3 . The patient developed a local failure at 9 months post-RT

illustrates local control stratified by pre-RT MRI risk profiles. At 2 years, the local control rate was approximately $73 \%$ in the low-risk group and $29 \%$ in the high-risk group, which was statistically significant $(95 \% \mathrm{CI}: 1.9-8.5$; $\mathrm{p}=0.0001)$.

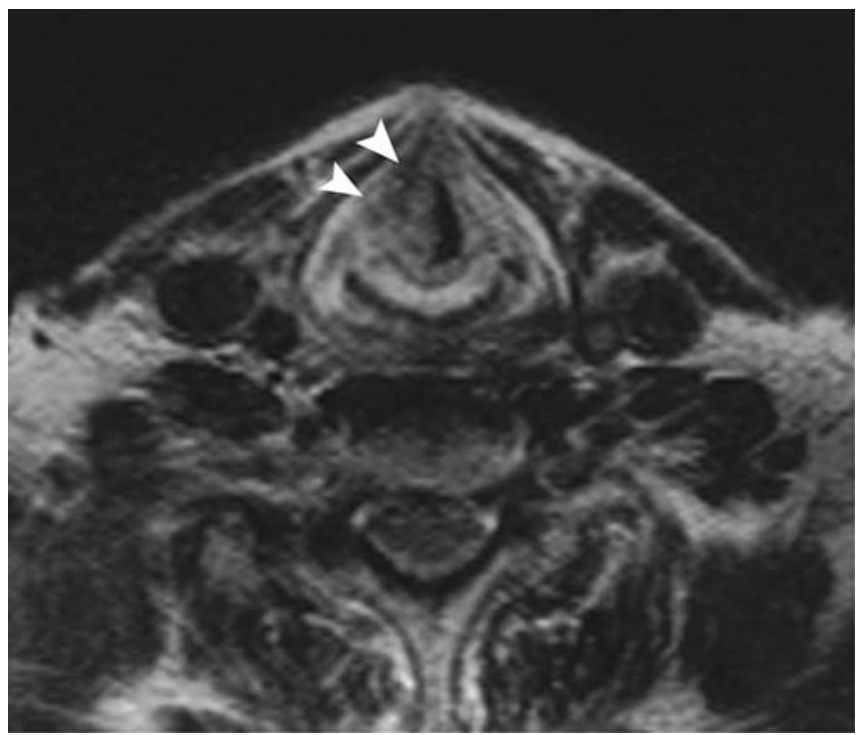

Fig. 5 Clinical stage T2 glottic carcinoma in a 78-year-old patient. Posttreatment axial T2-weighted MR image, obtained 5 months after RT, at the level of the true vocal cord demonstrates a tumor mass (arrowheads) with low to intermediate to high signal intensity. The tumor was considered as an isointense/mixed tumor, which locally recurred within 9 months after RT 


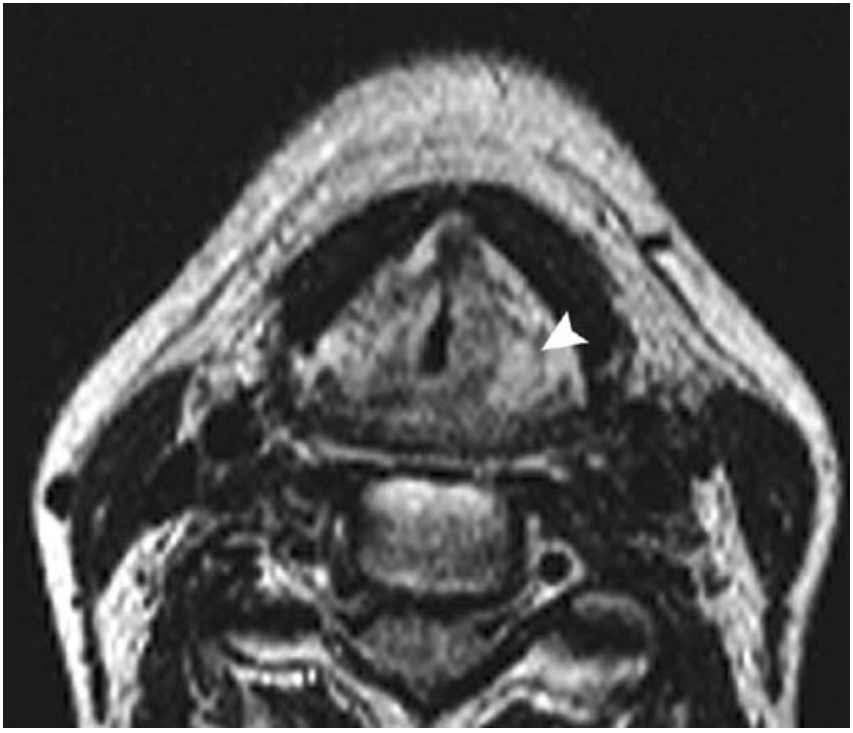

Fig. 6 Clinical stage T4 supraglottic carcinoma in a 65-year-old patient. Posttreatment axial T2-weighted MR image, obtained 3 months after RT, at the level of the false vocal cord shows a tumor mass (arrowheads) with high signal intensity. The tumor was considered as a hyperintense tumor, which locally recurred within 10 months after RT

\section{Post-RT MRI score}

The follow-up MR images of 80 patients were recorded by using a three-point, post-radiation therapy changes scale, and results were as follows: (1) score $=1$, i.e., 21 patients with the complete remission after radiotherapy, 33 patients with score 2, and 26 patients with MR imaging indicating definite local failure (score $=3$ ).

Figure 8 illustrates the local control stratified for post-RT MRI risk profiles. The local control rates at 2 years were $100 \%$ for score $1,64 \%$ for score 2 , and $4 \%$ for score 3 , which was statistically significant (95\% CI: 4.2-15.5; $\mathrm{p}<$ $0.0001)$. The sensitivity, specificity, accuracy, and negative and positive predictive values of use of follow-up MRI examination for detection of local failure (post-RT MRI score 3) were $96 \%(25 / 26), 76 \%(41 / 54), 83 \%(66 / 80)$, $98 \%(41 / 42)$, and $66 \%(25 / 38)$, respectively.

Combination of pre-RT MRI risk profile and post-RT MRI score

In the group of patients classified as a low pretreatment risk profile $(\mathrm{n}=38), 13$ patients had posttreatment score 1 and showed no local recurrence, whereas 8 patients with posttreatment score 3 all failed locally. In the group with posttreatment score $2(n=17), 4$ local recurrences occurred (24\%). Local control rates at 2 years were $100 \%$ for score $1,60 \%$ for score 2 , and $0 \%$ for score 3 (HR $7.3 ; 95 \%$ CI: 3.9-6330.9; $<<0.001)$.

In patients with a high pretreatment risk profile $(n=28)$, 3 patients had posttreatment score 1 and none of these cases developed a local recurrence, whereas for 15 patients with posttreatment score 3, 14 failed locally. In the group with posttreatment score $2(n=10)$, six local recurrences occurred $(60 \%)$. Local control rates at 2 years were $100 \%$ for score $1,40 \%$ for score 2 , and $7 \%$ for score 3 (HR 6.3; 95\% CI: $1.2-6.8 ; \mathrm{p}=0.01)$.

Post-RT MR imaging and local failure

Table 3 demonstrates the post-RT MRI examinations and disease status (failure vs. not failure). In 25 out of the 38 patients with local failure, the findings of the first follow-up MRI examination were classified as having a posttreatment score of 3. One patient without local failure had posttreatment score of 3 due to chondronecrosis and very pronounced edema at clinical examination treated with hyperbaric oxygen therapy. This patient remained free of local recurrence.

In 13 out of 38 patients with local failure, findings of first post-RT MRI examination were classified as a posttreatment score of 2. In four of these patients, an increase of the posttreatment MRI score from 2 to 3 occurred over time. In the other 4 of 13 patients, the follow-up MRI was only

Table 2 Pretreatment low-risk versus high-risk profile based MR imaging parameters

\begin{tabular}{lll}
\hline Site & Number of patients & $\%$ of total \\
\hline $\begin{array}{l}\text { Supraglottis } \\
\text { Low-risk }\end{array}$ & 14 & 17.5 \\
High-risk PES + abnormal SI of thyroid at ant.comissure & 6 & 7.5 \\
$\quad$ PES + abnormal SI of cricoid & 6 & 7.5 \\
No data avalaible & 6 & 7.5 \\
Glottis & 24 & 30 \\
Low-risk & 15 & 19 \\
High-risk Intermediate T2 signal in cartilage & 1 & 1 \\
Nypopharyngeal extension & 8 & 10
\end{tabular}

PES = pre-epiglottic space involvement; SI = signal intensity 


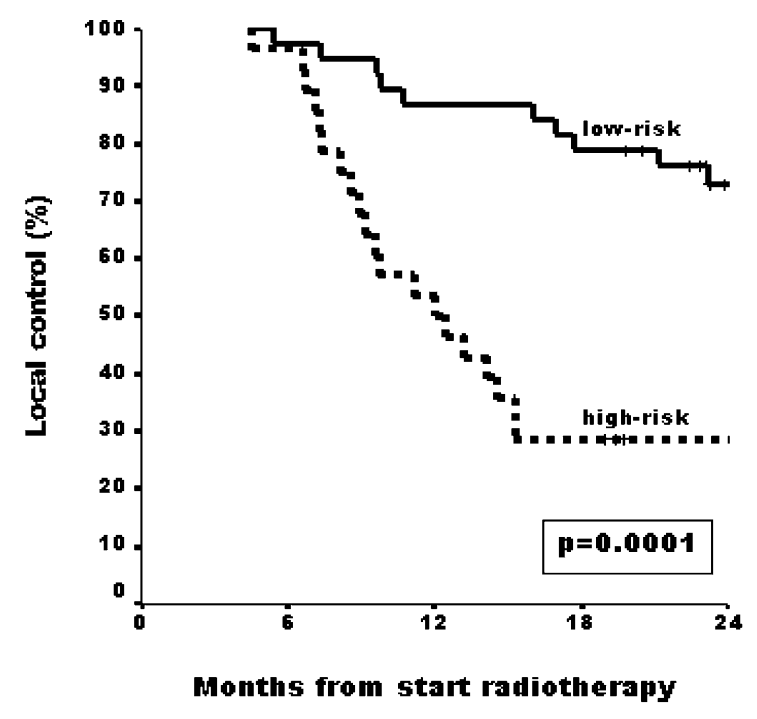

Straight line=Low-risk group (38 patients); Dotted line=High-risk group (28

patients). In 14 patients the pre-treatment risk profile was not available.

Fig. 7 Patients with a high pre-treatment MR risk profile showed poor prognosis (2-year local control rate: $29 \%$ ) compared to patients with a low pre-treatment MR risk profile (2-year local control rate: $73 \%$ )

performed 3-7 months after RT, and diagnosis of tumor recurrence was confirmed 1 to 5 months later.

None of the patients with a post-RT MRI score of $1 \mathrm{had}$ a tumor recurrence at the time of follow-up MR imaging.

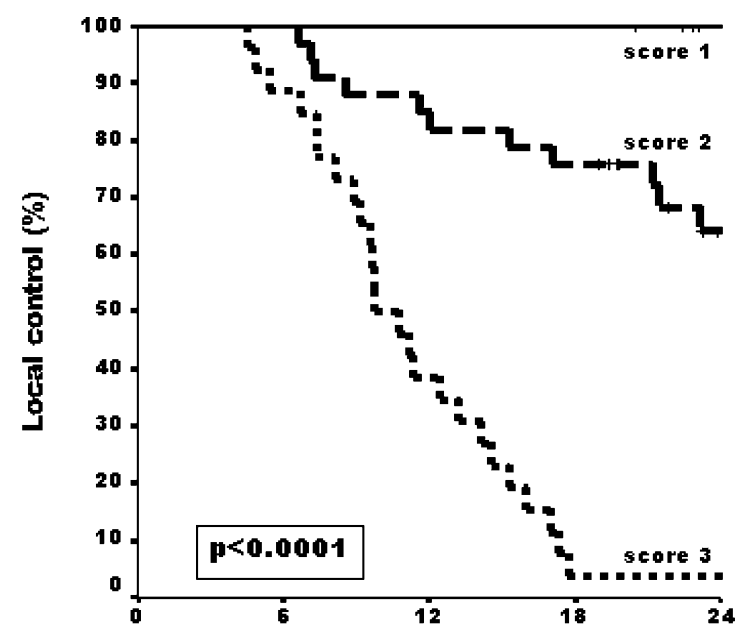

Wonths after completion of radiotherapy

Straight line=Score1: likely achieved permanent local control (21 patients); Interrupted line=Score 2: indeterminate (33 patients); Dotted line=Score 3 : suspicious for local failure ( 26 patients). Score 1 vs. Score 2: $p=0.9$; Score 1 vs. Score 3: $p<0.001$.

Fig. 8 Patients with a posttreatment MR risk profile of 3 showed clearly poor prognosis (2-year local control rate: $4 \%$ ) compared to patients with posttreatment MR risk profiles of 1 and 2 (2-year local control rate: $100 \%$ and $64 \%$, respectively)
Table 3 Detection of local recurrence and post-RT MRI examinations $(\mathrm{n}=80)$

\begin{tabular}{llll}
\hline Post-RT & No local recurrence & Local recurrence & \% of total \\
\hline Score 1 & 21 & 0 & 26 \\
Score 2 & 20 & 13 & 41 \\
Score 3 & 1 & 25 & 33 \\
\hline
\end{tabular}

Score 1 = likely achieved permanent local control; score $2=$ indeterminate; score 3 = suspicious for local failure

In addition, in 10 out of $38(26 \%)$ patients the local failure was first suspected on routine MR imaging (post-RT MRI score of 2 or 3). In six of these ten patients, these MRI findings were confirmed within 2 months by histopathologic examination. In the remaining four patients, no immediate action was undertaken (not within 4 months after radiologic diagnosis) because of the paucity of clinical symptoms or lack of suspicious findings on direct laryngoscopy.

From the entire patient population, 42 out of 80 patients were examined after irradiation with MRI between 1 and 10 months (mean, 3.8 months; median, 3.6 months), and these had no evidence of disease; in 25 out of 80 patients, the post-RT MR imaging showed clearly the residual disease suspected clinically with a mean delay of 7 months (median, 5.2 months). In the remaining 13 out of 80 patients, post-RT MR imaging did not indicate recurrent tumor, but these patients did develop a local failure with a mean delay for post-RT MRI examination of 5 months (median, 3.9 months). There was significant association between means of delay of post-MRI examination (test of variance, $\mathrm{p}=0.003$ ).

Observed lesions on posttreatment T2-weighted MR images

In T2-weighted MR images after radiation therapy, 35 tumors were hypointense $(44 \%), 25$ isointense/mixed $(31 \%)$, and 20 were hyperintense $(25 \%)$ compared with the signal intensity of the contralateral side of the larynx. From the 38 patients that recurred locally, 9 patients had hypointense tumor lesions (24\%), 12 patients had isointense $(32 \%)$, and finally 17 patients had hyperintense lesions (45\%). Figure 9 illustrates local control stratified by intensity on T2-weighted images after RT. At 2 years the local control rates were $77 \%$ for hypointense, $54 \%$ for isointense/mixed, and $15 \%$ for hyperintense lesions $(95 \%$ CI: $1.7-4.1 ; \mathrm{p}<0.001)$.

\section{Discussion}

Monitoring the response to non-surgical treatment is one of the challenges in head and neck oncology today. Accurate 


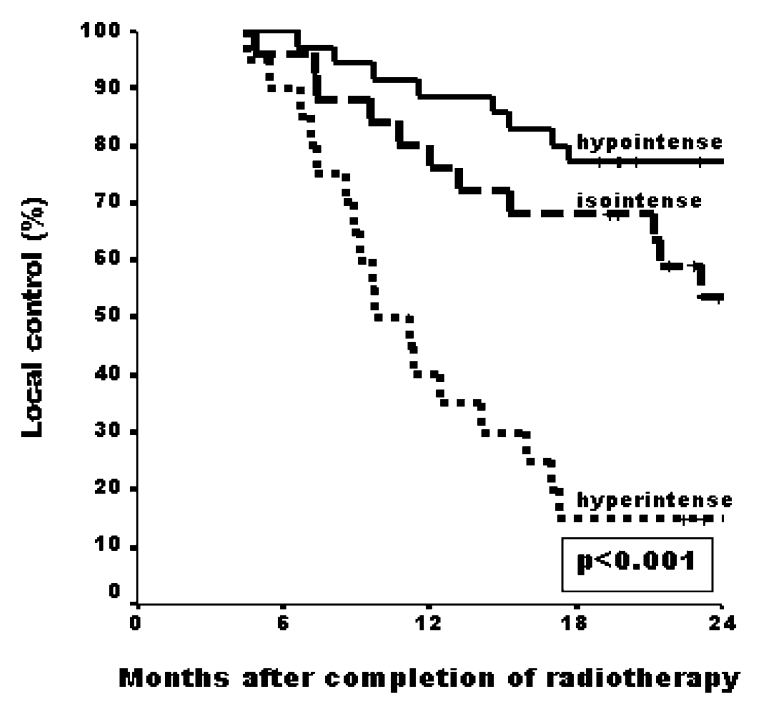

Straight line=Hypointense tumor mass (35 patients); Interrupted line=Isointense tumor mass (25 patients); Dotted line=Hyperintense tumor mass (20 patients). Hypointense vs. Isointense: $p=0.07$; Hypointense vs. Hyperintense: $p<0.001$.

Fig. 9 Patients with a hyperintense tumor mass on posttreatment T2-weighted MR image showed very bad prognosis (2-year local control rate: $15 \%$ compared to patients with a hypointense and isointense/mixed tumor mass on posttreatment T2-weighted MR image; 2-year local control rate: $77 \%$ and $54 \%$, respectively)

evaluation of the response to RT by clinical examination may be hampered by posttreatment fibrosis, edema, inflammation, or scarring. According our study, combined MR imaging before and after primary treatment may be helpful in evaluating response in laryngeal carcinoma treated by radiotherapy.

First, accurate pretreatment staging is essential for planning of optimal therapy for patients with laryngeal carcinoma, and data in this study provided valuable information for identifying patients at high risk for local recurrence. Around $42 \%$ of patients were retrospectively identified as the high-risk pretreatment MRI group, which means that the primary treatment chosen in these patients may not have been optimal.

In this study, recurrence after radiotherapy may be detected in an early stage on MR imaging. Especially the identification of patients who are at high risk (post-RT MRI score 3 ) for local failure was highly significant with regard to local control. Only 1 out of 26 patients in the posttreatment score 3 group remained free of disease at the primary site and showed osteoradionecrosis, which mimicked tumor recurrence. Our results are consistent with CT studies that report $10 \%$ local control rate at 2 years for patients in the score 3 group [15] and based on posttreatment $\mathrm{CT}$ findings a high significance of predicting the likelihood of local control in laryngeal carcinoma [1517]. Application of the post-RT MRI scoring system within the two pretreatment risk groups resulted in an additional separation within both groups into three risk groups for local recurrence. In patients with both a low and high pretreatment MRI risk profile, classification into the postRT MRI score 1 group was an infallible sign and proved to be strongly predictive for local control. Three patients classified as high risk on pre-RT MRI risk profile with a favorable response to RT were identified on post-RT MRI as a score 1 group and had no evidence of residual disease. Moreover, eight patients with initially low pretreatment MRI risk profile were identified as poor responders by follow-up MRI examination, and these patients recurred locally. Consequently, the post-RT MRI scoring system was demonstrated to be a better prognosticator than pretreatment MRI risk profile. Lell et al. [18] have reported that the main criteria for recurrent/residual tumor in MR imaging was infiltrative mass with intermediate signal intensity on T1-weighted, high signal intensity in T2weighted and enhancement after Gd-DTPA in T1-weighted MR images. They concluded that because of differences in aspect, MR imaging proved to be better than $\mathrm{CT}$ in characterizing recurrent lesions and in differentiation between scar tissue and local tumor failure. According to Engelbrecht et al. [20], the specificity of CT to detect recurrence was $76 \%$ and of MRI was $86 \%$. MRI is reported to be more sensitive $(92 \%)$ than CT $(81 \%)$.

The potential role of MR imaging in distinguishing tumor recurrence from radiation fibrosis on the basis of signal intensity on long repetition time (TR)/echo time (TE) (T2-weighted) pulse sequences for several head and neck carcinomas treated with radiotherapy has been described $[21,25,26]$. These studies conclude that tumors, that appeared hyperintense on T2-weighted MR images after radiation therapy were significantly correlated with pathologic findings $[25,26]$. In accordance with previous studies, our results suggest that recurrent tumors may appear as a hyperintense mass after contrast administration in comparison with fibrotic changes after RT, which can be seen as hypointense masses. However, the prolonged T2 value is not specific for tumor and may be seen in radiation edema or infection. Three patients with a hyperintense mass in our study proved to be false positive. Ebner et al. [21] found that MR imaging was useful in distinguishing recurrent tumors in the female pelvis from posttreatment fibrosis in six patients. They emphasized that signal intensities from radiation changes vary with time; fatty replacement changes are seen, and abnormal soft tissue that has decreased T2-weighted signal intensity is suggestive of posttreatment scarring rather than recurrent tumor $[18,19$, 27]. Arakawa et al. [28] also correlated MR imaging with pathology in nine patients with tongue cancer who underwent radiation or chemotherapy or both before surgery, and suggested that dynamic and T2-weighted MR imagings were considerably superior to contrastenhanced T1-weighted MR imaging in revealing tumor mass. Hyperintensity on T2-weighted MR imaging in 
recurrent tumors may be caused by increased intracellular water content of tumor cells and tumor edema.

CT findings of the laryngeal carcinoma within 3 to 6 months post-RT are reported to be often nonspecific [13, 14]. In our study the first MR study is performed 3-4 months after RT to evaluate the primary site, assuming no strong clinical suspicion of failure is present. MRI examination less than 4 months after the end of RT may be very helpful as a baseline, which may be useful to distinguish mass with complete remission. These findings suggest that in the posttreatment score 1 group, additional MRI follow-up is actually not necessary, unless there is clinical suspicion of recurrence. Moreover, the results of our study showed that recurrence followed later than 5 months (median value) after RT were detectable more easily on MR imaging than tumor recurrence earlier than 4 months after RT. In accordance with our results, Lell et al. [18] reported that in the first 34 months, edema, tumor necrosis, and acute or chronic inflammation lead to signal changes equivalent to tumor, but with long intervals, these changes are minimized and diagnosis is becoming more sufficient. Therefore, the use of MR imaging for assessment of tumor recurrence within 4 months after the end of radiation therapy may not be indicated. First post-RT MRI examination should be performed at 4 months after the end of RT. Progression of pathologic findings is strongly predictive of recurrence, such that an early MR imaging might lead to earlier detection of recurrence than those of clinical examination ( $26 \%$ of local failure in this study). Patients with "suspicious" post-RT MR study should be followed by imaging at regular intervals later than 4 months, and therefore this might help any urge to biopsy prematurely. This approach is supported by data from FDG-positron emission tomography (PET) studies, showing that baseline exams before 4 months post-RT may not accurately reflect outcome, and that studies done at 4 months and later result in a more accurate prediction of outcome [29, 30]. Keane et al. [31] supported routine reassessment with biopsy 8-12 weeks after completion of radiation therapy to detect recurrence as early as possible. Results of this study suggest that use of post-RT MRI examination could reduce substantially the number of patients needing biopsy.
The follow-up imaging of laryngeal carcinoma patients treated with chemoradiotherapy generally shows no obvious difference from those treated with radiation therapy alone. It is important to note that, in this retrospective setting, no strict follow-up protocol with repeat MR studies was followed.

Traditionally, the measurement of tumor volume has been widely used for evaluating response after oncologic therapy, and correlation with tumor control has been documented [32-34]. We did not include MR volumetric tumor evaluation after RT in our study due to factors contributing to apparent tumor radioresistance; it was very difficult to outline real tumor margins. Within the limitations of this retrospective study, it can be concluded that the manual drawing of the ROI area may result in some errors related to the operator's experiences and obscure the tumor margin. Although our study is limited by no standard time for followup of patients after primary treatment and subjective criteria for the diagnosis of radiation change versus recurrent tumor (observed lesions on T2 images), there was good intraobserver and interobserver agreement.

\section{Conclusion}

In the evaluation of laryngeal carcinoma on post-RT MRI examinations, symmetric post-RT changes without detectable mass are strongly indicative of ultimate control at the primary site. When post-RT MRI shows a discrete mass or asymmetric post-RT changes at the primary site, the pre-RT MRI high-risk profile and hyperintense mass on T2weighted images after radiation therapy should be suggestive for further workup in patients. Follow-up MR study performed 4 months or later after RT appears to be a reliable tool to refer a residual mass to scar tissue and is recommended in addition to careful clinical follow-up.

Open Access This article is distributed under the terms of the Creative Commons Attribution Noncommercial License which permits any noncommercial use, distribution, and reproduction in any medium, provided the original author(s) and source are credited.

\section{References}

1. Abdel Razek AA, Kandeel AY, Soliman N, El-shenshawy HM, Kamel Y, Nada N, Denewar A (2007) Role of diffusionweighted echo-planar MR imaging in differentiation of residual or recurrent head and neck tumors and posttreatment changes. AJNR Am J Neuroradiol 28:1146-1152
2. Ljumanovic R, Langendijk JA, Schenk $\mathrm{B}$, Van Wattingen M, Knol DL, Leemans CR, Castelijns JA (2004) Supraglottic carcinoma treated with curative radiation therapy: identification of prognostic groups with MR imaging. Radiology 232:440-448

3. Isaacs JH, Mancuso AA, Mendenhall WM, Parsons JT (1988) Deep spread patterns in CT staging of T2-4 squamous cell laryngeal carcinoma. Otolaryngol Head Neck Surg 99: 455-464
4. Hermans R, Van den Bogaert W, Rijnders A, Baert AL (1999) Value of computed tomography as outcome predictor of supraglottic squamous cell carcinoma treated by definitive radiation therapy. Int J Radiat Oncol Biol Phys 44:755-765

5. Pameijer FA, Balm AJM, Hilgers FJM, Muller SH (1997) Variability of tumor volumes in T3-staged head and neck tumors. Head Neck 19:6-13 
6. Mukherji SK, O'Brien SM, Gerstle RJ, Weissler M, Shockley W, Stone JA, Castillo M (2000) The ability of tumor volume to predict local control in surgically treated squamous cell carcinoma of the supraglottic larynx. Head Neck 22:282-287

7. Freeman DE, Mancuso AA, Parsons JT, Mendenhall WM, Million RR (1990) Irradiation alone for supraglottic larynx carcinoma: can CT findings predict treatment results? Int J Radiat Oncol Biol Phys 19:485-490

8. Mancuso AA, Mukherji SK, Schmalfuss I, Mendenhall W, Parsons J, Pameijer F, Hermans R, Kubilis P (1999) Preradiotherapy computed tomography as a predictor of local control in supraglottic carcinoma. J Clin Oncol 17:631-637

9. Hermans R, Feron M, Bellon E, Dupont $\mathrm{P}$, Van den Bogaert W, Baert AL (1998) Laryngeal tumor volume measurements determined with CT: a study on intra- and interobserver variability. Int J Radiat Oncol Biol Phys 40:553-557

10. Kraas JR, Underhill TE, D'Agostino RB Jr, Williams DW, Cox JA, Greven KM (2001) Quantitative analysis from $\mathrm{CT}$ is prognostic for local control of supraglottic carcinoma. Head Neck 23:1031-1036

11. Ljumanovic R, Langendijk JA, van Wattingen M, Schenk B, Knol DL, Leemans CR, Castelijns JA (2007) MR imaging predictors of local control in glottic squamous cell carcinoma treated with radiation alone. Radiology 244:205-212

12. O'Brien PC (1996) Tumour recurrence or treatment sequela following radiotherapy for larynx cancer. J Surg oncol 63:130-135

13. Mukherji SK, Mancusso AA, Kotzur IM, Mendenhall WM, Kubilis PS, Tart RP, Lee WR, Freeman D (1994) Radiologic appearance of the irradiated larynx. Part I. Expect changes. Radiology 193:141-148

14. Mukherji SK, Mancusso AA, Kotzur IM, Mendenhall WM, Kubilis PS, Tart RP, Freeman D, Lee WR (1994) Radiologic appearance of the irradiated larynx. Part II. Primary site response. Radiology 193:149-154

15. Pameijer FA, Hermans R, Mancuso AA, Mendenhall WM, Parsons JT, Stringer SP, Kubilis PS, Van Tinteren H (1999) Pre- and post-radiotherapy computed tomography in laryngeal cancer: imaging-based prediction of local failure. Int J Radiat Oncol Biol Phys 45:359-366
16. Hermans R, Pameijer FA, Mancuso AA, Parsons JT, Mendenhall WM (2000) Laryngeal or hypopharyngeal squamous cell carcinoma: can followup CT after definitive radiation therapy be used to detect local failure earlier than clinical examination alone? Radiology 214:683-687

17. Ojiri H, Mendenhall WM, Mancuso AA (2002) CT findings at the primary site of oropharyngeal squamous cell carcinoma within 6-8 weeks after definitive radiotherapy as predictors of primary site control. Int J Radiat Oncol Biol Phys 52:748-754

18. Lell M, Baum U, Greess H, Nömayr A, Nkenke E, Koester M, Lenz M, Bautz W (2000) Head and neck tumors: imaging recurrent tumor and posttherapeutic changes with CT and MRI. Eur J Radiol 33:239-247

19. Glazer HS, Niemeyer JH, Balfe DM, Hayden RE, Emami B, Devineni VR, Levitt RG, Aronberg DJ, Ward MP, Lee JKT, Sagel SS (1986) Neck neoplasmas: MR imaging. Part II. Posttreatment evaluation. Radiology 160 : 349-354

20. Engelbrecht V, Pisar E, Fürst G, Mödder U (1995) Follow-up and diagnosis of malignant head and neck tumors after radiochemotherapy. Comparison of computed tomography and magnetic resonance tomography. Rofo Fortschr Geb Röntgenstr Neuen Bildgeb Verfahr 162:304-310

21. Gong QY, Zheng GL, Zhu HY (1991) MRI differentiation of recurrent nasopharyngeal carcinoma from postradiation fibrosis. Comput Med Imag graph $15: 423-429$

22. Kabala J, Goddard P, Cook P (1992) Magnetic resonance imaging of extracranial head and neck tumours. Br J Radiol 65:375-383

23. Sobin LH, Wittekind Ch (2002) TNM classification of malignant tumors, 6th edn. International Union Against Cancer, Wiley-Liss, New York, pp 36-42

24. Mukherji SK, Wolf GT (2003) Evaluation of head and neck squamous cell carcinoma after treatment. AJNR Am J Neuroradiol 24:1743-1746

25. Minnetti M, Misiti A, Palmeggiani F, Iacari V, Macori F, Anaveri G (1996) Assessment with magnetic resonance imaging of response to radiotherapy for tongue and mouth floor tumors. Radiol Med 92:624-628

26. Tomura N, Watanabe O, Kato K, Takahashi S, Watarai J, Sageshima M, Yokomizo M (2002) Irradiated carcinoma of the tongue: correlation of MR imaging findings with pathology. AJR Am J Roentgenol 178:705-710
27. Ebner F, Kressel HY, Mintz MC, Carlson JA, Cohen EK, Schiebler M, Gefter W, Axel L (1988) Tumor recurrence versus fibrosis in the female pelvis: differentiation with MR imaging at $1.5 \mathrm{~T}$. Radiology $166: 333-340$

28. Arakawa A, Tsuruta J, Nishimura R, Sakamoto Y, Korogi Y, Baba Y, Furusawa M, Ishimaru Y, Uji Y, Taen A, Ishikawa T, Takahashi M (1996) Lingual carcinoma: correlation of MR imaging with histopathological findings. Acta Radiol 37:700-707

29. Greven KM, Williams DW, Keyes JW, McGuirt WF, Harkness BA, Watson NE, Raben M, Frazier LC, Geisinger KR, Cappellari JO (1994) Distinguishing tumor recurrence from irradiation sequelae with positron emission tomography in patients treated for larynx cancer. Int J Radiat Oncol Biol Phys 29:841-845

30. Greven KM, Williams DW, Keyes JW, McGuirt WF, Watson NE, Randall ME, Raben M, Geisinger KR, Cappellari JO (1994) Positron emission tomography of patients with head and neck carcinoma before and after high dose irradiation. Cancer 74:1355-1359

31. Keane TJ, Cummings BJ, O'Sulivan B, Payne D, Rawlinson E, MacKenzie R, Danjoux C, Hodson I (1993) A randomized trial of radiation therapy compared to split course radiation therapy combined with mitomycin $\mathrm{C}$ and 5 fluorouracil as initial treatment for advanced laryngeal and hypopharyngeal squamous carcinoma. Int J Radiat Oncol Biol Phys 25:613-618

32. Mayr NA, Magnotta VA, Ehrhardt JC, Wheeler JA, Sorosky JI, Wen BC, Daris CS, Pelsang RE, Anderson B, Doornbos JF, Hussey DH, Yuh WT (1996) Usefulness of tumor volumetry by magnetic resonance imaging in assessing response to radiation therapy in carcinoma of the uterine cervix. Int J Radiat Oncol Biol Phys 35:915-924

33. Johnson CR, Khandelwal SR, SchmidtUllrich RK, Ravalese J, Wazer DE (1995) The influence of quantitative tumor volume measurements on local control in advanced head and neck cancer using concomitant boost accelerated superfractionated irradiation. Int J Radiat Oncol Biol Phys 32:635-641

34. Ohno Y, Kusumoto M, Kono M (1997) Evaluation of therapeutic effect using enhanced MRI in lung cancer. Evaluation of methods in terms of necrosis. Nippon Igaku Hoshasen Zasshi 57:783-790 